\title{
A New Model of NGC 6210 to Solve its Abundance Discrepancy Problem
}

\author{
V. Escalante ${ }^{1}$ and J. A. Toalá ${ }^{2}$ \\ ${ }^{1}$ Instituto de Radioastronomía y Astrofísica \\ UNAM, Morelia 58341, Michoacán, México \\ email: v.escalante@crya. unam.mx \\ ${ }^{2}$ Institute of Astronomy and Astrophysics \\ Academia Sinica (ASIAA), Taipei 10617, Taiwan \\ email: toala@asiaa.sinica.edu.tw
}

\begin{abstract}
We present a model that solves the abundance discrepancy problem for NGC 6210 . The model proposes a high abundance of CNONe elements that lowers the temperature of the central parts of the nebula. The colder gas model reproduces the observed intensity of the strong $[\mathrm{N}$ II] and [O III] emission lines, and increases the predicted weak recombination lines towards their observed values. We examine how the usual nebular diagnostic line ratios depend on model abundances.
\end{abstract}

Keywords. ISM: abundances, planetary nebula: NGC 6210

The discrepancy between abundances derived from collisionally excited lines (CELs) and from optical recombination lines (ORLs) is a long standing conundrum in the theory of interstellar nebulae. Usually ORL derived abundances are larger than CEL derived abundances. A model optimized to reproduce CEL intensities calculated by Bohigas et al. (2015) also shows an abundance discrepancy in the sense that it underestimates ORL intensities because the predicted $\mathrm{O}^{+2}$ column density is too low. We used the code CLOUDY (Ferland et al. 2013) to construct new chemically homogeneous models of NGC 6210 with increased CNONe abundances to reproduce CELs and ORLs simultaneously.

The models propose a binary star with $T_{\text {eff }}=179300 \mathrm{~K}, L=1635 L_{\odot}, \log g=$ 7.0, $T_{\text {eff }}=29700 \mathrm{~K}, L=1694 L_{\odot}, \log g=4.5$ as suggested by the relation between binarity and the abundance discrepancy problem (Corradi et al. 2015). The density profile was chosen to reproduce the observed intensities of [N II], [O II], and [O III] optical CELs, the [O III] $51.80 \mu \mathrm{m}$, and the He I $5876 \AA$ and He II $4686 \AA$ lines reported by Bohigas et al. (2015) and Pottasch et al. (2009). The density profile has a tail that decreases with radius as $r^{-4}$ to produce the extended halo of the nebula.

The $\mathrm{O}$ abundance in the new model, $\mathrm{O} / \mathrm{H}=1.33 \times 10^{-3}$, was chosen to reproduce the $\mathrm{O}$ II ORLs lines to eliminate the abundance discrepancy. The model produces an inner zone with temperatures as low as $6600 \mathrm{~K}$ due to the higher $\mathrm{O}^{+2}$ concentration, where the ORL emission is produced (see Fig. 1). The temperature rises at the edges of the $\mathrm{O}^{+2}$ zone thus increasing the [O III] optical emission, and producing a temperature gradient with a Peimbert parameter $t^{2}=0.04$. Images obtained by García-Rojas, et al . (2016) of another nebula with a high abundance discrepancy, NGC 6778, show a similar concentration of ORL oxygen emission inside the CEL oxygen emission.

The model calculated by Bohigas et al. (2015) with a lower $\mathrm{O} / \mathrm{H}$ abundance uses a density profile varying as $r^{2}$. A comparison with observations in Table 1 shows that both models reproduce the lines to comparable accuracy, except for the [O II] emission. The 

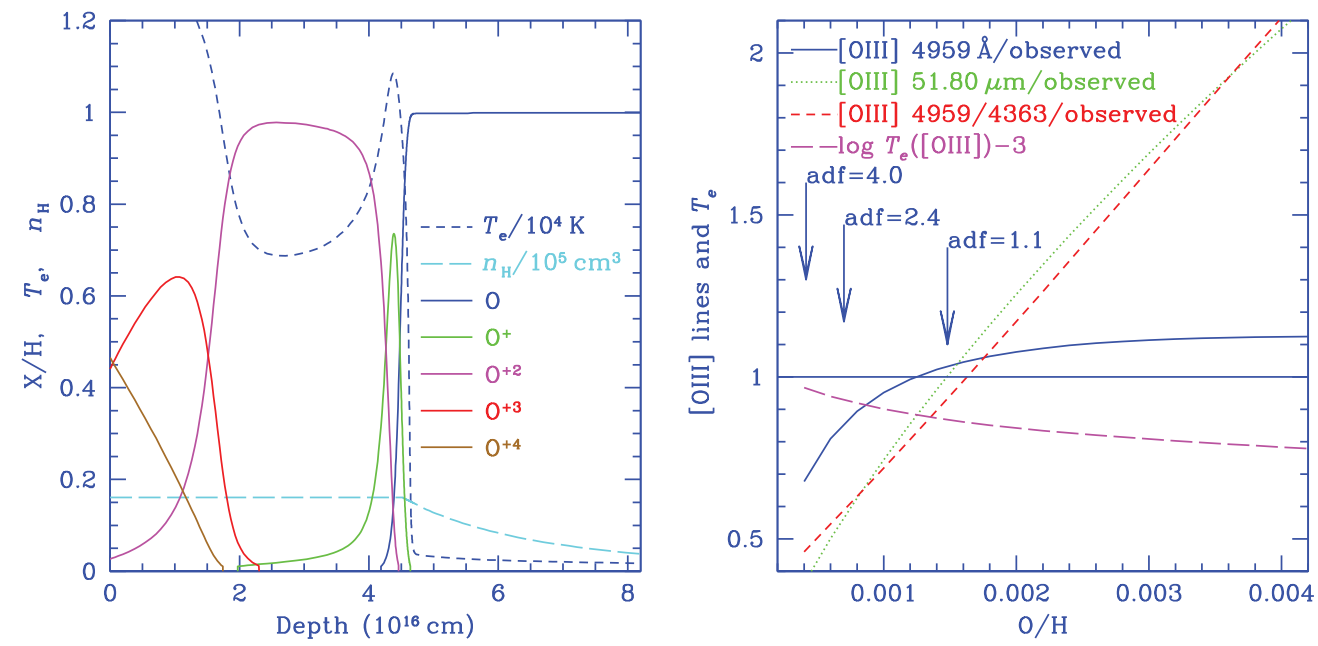

Figure 1. O ionization, electron temperature and density structure (left), and [O III] line intensities vs. $\mathrm{O}$ abundance (right) in our new model.

Table 1. A few comparisons of predicted and observed line intensities.

\begin{tabular}{|c|c|c|c|c|c|c|c|c|c|c|c|}
\hline $\begin{array}{l}\text { Model } \\
\& \text { Obs }\end{array}$ & $\begin{array}{c}\mathrm{O} / \mathrm{H} \\
\left(10^{-4}\right)\end{array}$ & $\mathrm{adf}$ & $\begin{array}{l}{\left[\begin{array}{ll}\mathrm{N} & \mathrm{II}\end{array}\right]} \\
6584\end{array}$ & $\begin{array}{l}{[\mathrm{N} \text { II] ratio }} \\
6584 / 5755\end{array}$ & $\begin{array}{l}{\left[\begin{array}{ll}\mathrm{O} & \mathrm{II}\end{array}\right]} \\
3726\end{array}$ & $\begin{array}{c}{\left[\begin{array}{ll}\mathrm{O} & \mathrm{III}\end{array}\right]} \\
4959\end{array}$ & $\begin{array}{c}{[\mathrm{O} \text { III] ratio }} \\
4959 / 4363\end{array}$ & $\begin{array}{l}{\left[\begin{array}{ll}\mathrm{O} & \mathrm{III}\end{array}\right]} \\
51.80\end{array}$ & 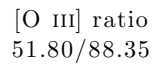 & $\begin{array}{l}\text { O II } 4649 \\
\text { Mult V1 }\end{array}$ & $\begin{array}{l}\text { O II } 4349 \\
\text { Mult V2 }\end{array}$ \\
\hline $\mathrm{Obs}^{1}$ & 4.10 & 4.0 & 15.68 & 54.4 & 18.33 & 343.40 & 58.3 & $151^{4}$ & $3.17^{4}$ & 0.446 & 0.113 \\
\hline $\mathrm{Obs}^{2}$ & 4.36 & 3.1 & 18.70 & 47.6 & 24.95 & 361.40 & 59.5 & & & 0.389 & 0.120 \\
\hline $\begin{array}{l}\text { Low } \mathrm{O} \\
\text { model }^{1}\end{array}$ & 7.42 & 2.4 & 15.69 & 22.4 & 18.95 & 343.00 & 59.8 & 26.0 & 8.69 & 0.322 & 0.130 \\
\hline $\begin{array}{l}\text { High O } \\
\text { model }^{3}\end{array}$ & 13.3 & 1.1 & 15.68 & 43.4 & 48.45 & 332.69 & 61.4 & 53.3 & 8.19 & 0.423 & 0.169 \\
\hline
\end{tabular}

${ }^{1}$ Bohigas et al. (2015), ${ }^{2}$ Liu et al. (2004), ${ }^{3}$ This work, ${ }^{4}$ Pottasch et al. (2009)

[O II] emission appears asymmetrical in HST archival images (Guerrero et al. 2013), and therefore it is overestimated by our spherically symmetric model.

Figure 1 shows that the [O III] 4958 and $5007 \AA$ lines vary little at high O abundances because of the decrease in electron temperature while the [O III $51.80 \mu \mathrm{m}$ does not depend on temperature. The abundance discrepancy factor adf $=(\mathrm{O}$ abundance from ORLs $) /$ (O abundance from CELs) is given for the observed line intensities (4.0), the model abundance of Bohigas et al. (2015) (2.4), and the model with high O abundance (1.1).

\section{References}

Bohigas, J., Escalante, V., Rodríguez, M., \& Dufour, R. J. 2015, MNRAS, 447, 817

Corradi, R. L. M, García-Rojas, J., Jones, D., \& Rodríguez-Gil, P. 2015, Ap. J. 803, 99

Ferland, G. J., Porter, R. L., van Hoof, P. A. M., Williams, R. J. R., Abel, N. P., Lykins, M. L., Shaw, G., Henney, W. J., \& Stancil, P. C. 2013, RMxAA, 49, 137

García-Rojas, J., Corradi, R. L. M., Monteiro, H., Jones, D., Rodríguez-Gil, P., \& CabreraLavers, A. 2016, Ap. J. Lett, 824, L27

Guerrero, M. A., Toalá, J. A., Medina, J. J., Luridiana, V., Miranda, L. F., Riera, A., \& Velázquez, P. F. 2013, A\&\&A, 557, A121

Liu, Y., Liu, X.-W., Luo, S.-G., \& Barlow, M. J. 2004, MNRAS, 353, 1231

Pottasch, S. R., Bernard-Salas, J., \& Roellig, T. L. 2009, A\& A, 499, 249 